\title{
Precise Measurement of Semiconductor Laser Chirp Using Effect of Propagation in Dispersive Fiber and Application to Simulation of Transmission Through Fiber Gratings
}

\author{
Eva Peral, Member, IEEE, William K. Marshall, Member, OSA, \\ and Amnon Yariv, Life Fellow, IEEE, Fellow OSA
}

\begin{abstract}
Measurements of small-signal intensity modulation from direct-modulated distributed feedback (DFB) semiconductor lasers after propagation in dispersive fiber have previously been used to extract intrinsic laser chirp parameters such as linewidth enhancement factor and crossover frequency. Here, we demonstrate that the simple rate equations do not satisfactorily account for the frequency response of real DFB lasers and describe some experimental techniques that conveniently determine the precise laser chirp. Implications for simulation of high-speed lightwave systems are also considered.
\end{abstract}

Index Terms - Distributed feedback (DFB) lasers, gratings, lasers, laser measurements, optical fiber communication, optical fiber measurement applications, optical modulation, quantumwell lasers, semiconductor.

\section{INTRODUCTION}

$\mathbf{T}$ HE performance of high-speed lightwave systems using $1.55 \mu \mathrm{m}$ distributed feedback (DFB) semiconductor lasers with direct modulation is significantly affected by laser chirp, the frequency modulation (FM) which accompanies the intensity modulation (IM). Semiconductor laser chirp is commonly characterized by two parameters, the linewidth enhancement factor and the cross-over frequency between the adiabatic and transient regimes of chirp [1], which follow from a rate equation analysis of the laser.

Important effects that are not considered explicitly in simple laser rate equation analyses of laser chirp include the longitudinal and transverse spatial variations of the optical intensity and/or carrier density. Roughly speaking, these contribute to adiabatic chirp. Sophisticated, mostly numerical, analyses of the dynamic characteristics of multiquantum-well distributed feedback (MQW-DFB) lasers considering spatial hole burning, carrier transport, and carrier capture exist in the literature [2]-[8] and it is possible to infer from these analyses that in a laser the adiabatic chirp is, in fact, not quite adiabatic.

Manuscript received March 17, 1998; revised July 10, 1998. This work was supported by the Defense Advanced Research Projects Agency, the Office of Naval Research, and the Air Force Office of Scientific Research.

The authors are with the Department of Applied Physics, California Institute of Technology, Pasadena, CA 91125 USA.

Publisher Item Identifier S 0733-8724(98)07399-X.
Recently, it has been demonstrated that the frequency modulation to intensity modulation (FM-to-IM) conversion effect due to propagation in dispersive optical fiber can be used to measure conveniently the linewidth enhancement factor and crossover frequency of the laser [10]-[14]. In careful examination of data we have obtained with this technique, however, we have found that although a good fit to theory (and hence a small variance for the parameters) can be obtained when fitting results for a single length of fiber, for some lasers a large variation of the parameters can occur for results obtained with different lengths of fiber. We attribute this variation for different fibers to the more detailed dependence of laser chirp on modulation frequency that is implicit to a more detailed laser model.

In this paper, we demonstrate a new measurement technique that conveniently determines the precise characteristics of the laser chirp using the effect of propagation in optical fiber. The laser chirp thus measured can be directly compared to any of the more complete laser models cited above and, in addition, is shown here to lead to an improved agreement between experiment and theory in an optical transmission experiment involving fiber Bragg gratings.

In Section II, the small-signal baseband intensity transfer function of an arbitrary optical filter is derived. In Section III, it is demonstrated that the simple rate equation model can fail to accurately characterize the laser chirp of semiconductor lasers. Our technique for measuring the precise laser chirp using an optical fiber is then presented. In Section IV, the implications of the frequency dependence of laser chirp in simulation of transmission through fiber gratings are considered.

\section{THEORY}

A direct method for determining the laser chirp is to observe the sidebands of the optical field spectrum using an interferometer (see the Appendix). A more convenient method, however, is to introduce some optical filtering to convert part of the frequency modulation (FM) to intensity modulation (IM) so that it can be photodetected. Propagation through dispersive optical fiber can be used to produce this filtering [10]-[14].

The complex electric-field amplitude, $E$, at the output of a semiconductor laser directly modulated with a small-signal 
modulation frequency $\Omega /(2 \pi)$ can be expressed in the form:

$$
\begin{aligned}
E= & E_{0}\left[1+m_{\mathrm{IM}} \cos \left(\Omega t+\varphi_{\mathrm{IM}}\right)\right]^{1 / 2} \\
& \cdot \exp \left[i m_{\mathrm{FM}} \cos \left(\Omega t+\varphi_{\mathrm{FM}}\right)\right] \\
= & E_{0}\left[1+\left(\frac{\Delta P}{2 P_{0}} \exp (i \Omega t)+\text { c.c. }\right)\right]^{1 / 2} \\
& \cdot \exp [(i / 2)(\Delta \phi \exp (i \Omega t)+\text { c.c. })]
\end{aligned}
$$

where $m_{\mathrm{IM}}$ and $m_{\mathrm{FM}}$ are the IM and FM indexes, respectively. The ratio $\Delta \phi /\left(\Delta P / P_{0}\right)$ which for light produced by a semiconductor laser is a function of the modulation frequency, $\Omega$, will be referred to in what follows as the phase to intensity (modulation index) ratio $\operatorname{PIR}(\Omega)$.

In the small-signal regime, the electric field after propagation through an optical filter with transfer function $\tilde{H}(\omega)$ is given by

$$
\begin{aligned}
E= & E_{0}\left[1+\left(\frac{\Delta P}{2 P_{0}}+i \Delta \phi\right) \tilde{H}\left(\omega_{0}+\Omega\right) \exp (i \Omega t)\right. \\
& \left.+\left(\frac{\Delta P^{*}}{2 P_{0}}+i \Delta \phi^{*}\right) \tilde{H}\left(\omega_{0}+\Omega\right) \exp (-i \Omega t)\right]
\end{aligned}
$$

where $\omega_{0}$ is the laser optical frequency. The photodetected current is proportional to the square of the electric field amplitude. By squaring (2) and keeping linear terms in the modulation frequency, $\Omega$, the baseband small-signal transfer function, $H(\Omega)$, which is defined as the complex amplitude of the photocurrent at frequency $\Omega$ that will be detected after propagation through an optical filter normalized by that which would be detected at the laser output, is obtained as

$$
H(\Omega)=H_{e}(\Omega)+2 i H_{o}(\Omega) \operatorname{PIR}(\Omega)
$$

where $H_{e}(\Omega)$ and $H_{o}(\Omega)$ are the conjugate-symmetric and conjugate-antisymmetric parts of $\tilde{H}(\omega) \tilde{H}^{*}\left(\omega_{0}\right)$ around $\omega_{0}$. That is

$$
\begin{aligned}
& H_{e}(\Omega)=\frac{1}{2}\left[\tilde{H}\left(\omega_{0}+\Omega\right) \tilde{H}^{*}\left(\omega_{0}\right)+\tilde{H}^{*}\left(\omega_{0}-\Omega\right) \tilde{H}\left(\omega_{0}\right)\right] \\
& H_{o}(\Omega)=\frac{1}{2}\left[\tilde{H}\left(\omega_{0}+\Omega\right) \tilde{H}^{*}\left(\omega_{0}\right)-\tilde{H}^{*}\left(\omega_{0}-\Omega\right) \tilde{H}\left(\omega_{0}\right)\right]
\end{aligned}
$$

where $\tilde{H}(\omega)$ is the transfer function of the optical filter.

Equation (3) indicates that, in addition to the PIR of the laser, the dispersion and the asymmetry of the optical filter around the laser frequency $\omega_{0}$ determine the extent of FMto-IM conversion. In the case of dispersive fiber, the optical transfer function can be approximated by $\tilde{H}\left(\omega_{0}+\Omega\right)=$ $\exp \left[-i \beta_{1} \Omega z+i \theta(\Omega, z)\right]$, with $\theta(\Omega, z)=-\frac{1}{2} \beta_{2} \Omega^{2} z$. Here, $\beta_{1}$ is the inverse of the group propagation velocity, $\beta_{2}$ is the group velocity dispersion parameter and $z$ is the fiber length. Substitution in (3) and (4) yields for the baseband transfer function in this case

$$
H(\Omega, z)=[\cos \theta-2 \sin \theta \operatorname{PIR}(\Omega)] \exp \left(-i \beta_{1} \Omega z\right) .
$$

The simple rate equation model of semiconductor lasers [1] predicts a PIR in the small-signal regime of the form

$$
P I R \equiv \frac{\Delta \phi}{\Delta P / P_{0}}=-\frac{\alpha}{2}\left(1+\frac{\kappa}{i \Omega}\right)
$$

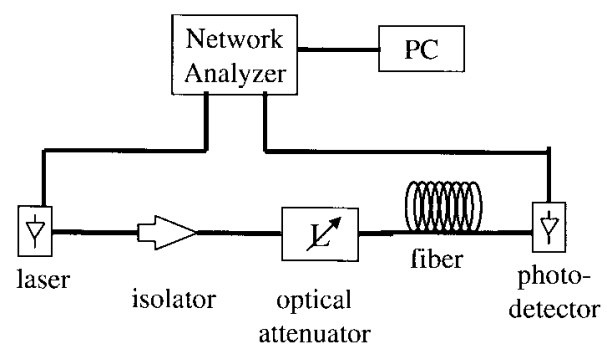

Fig. 1. Experimental setup.

where $\alpha$ is the linewidth enhancement factor and $\kappa$ is the crossover frequency between adiabatic and transient chirp regimes. $\kappa$ is related to the gain compression parameter, $\varepsilon$, and the photon lifetime, $\tau_{\mathrm{ph}}$, and $P_{0}$ via $\kappa=\left(\varepsilon P_{0} / \tau_{\mathrm{ph}}\right)$.

For a more detailed laser model [2]-[8], (6) is only approximately correct. The PIR can then be expressed, in general, as in (6), but with $\kappa$ replaced by a complex function of the modulation frequency, $\kappa(\Omega)=\kappa_{r}(\Omega)+i \kappa_{i}(\Omega)$. Equation (6) with $\kappa=\kappa(\Omega)$, however, is overdetermined in the sense that for any given value of $\alpha$, some complex function $\kappa(\Omega)$ can be found that describes the PIR. Another possibility would be to allow $\alpha=\alpha(\Omega)$ and $\kappa=\kappa_{r}(\Omega)$ [9] (an imaginary part for $\kappa(\Omega)$ is not needed in this case). However, the expression above is employed because, by using an appropriate theoretical model for the laser chirp, the value of $\alpha$ can be seen to be equal to that obtained from linewidth measurements, as will be further discussed elsewhere.

\section{MEASUREMENT}

\section{A. Simple Measurement of Laser Chirp}

The baseband transfer function, $H(\Omega, z)$, after propagation in a length $z$ of dispersive fiber, can be determined experimentally by measuring the modulation response after propagation in the fiber and normalizing it by the modulation response at the laser output. The experimental setup is shown in Fig. 1. The light of a directly modulated laser was coupled to the optical fiber and was passed through an isolater and then a variable optical attenuator. The optical power was attenuated so that the maximum power launched into the fiber was 2 $\mathrm{mW}$. It was checked that this attenuation was enough to avoid nonlinear effects in the modulation response. After propagation in fiber, the light was photodetected and then the phase and magnitude of the modulation response was measured using a network analizer. Using (5) and (6), the parameters $\alpha$ and $\kappa$, together with $\beta_{2}$ if it is unknown, can be found [10]-[14] by least squares fitting $|H(\Omega, z)|^{2}$. However, in our experience, this procedure can yield very different values for $\kappa$ and also to a lesser extent for $\alpha$ for different lengths of fiber.

Fig. 2 shows the change in modulation response, $|H(\Omega, z)|^{2}$, after propagation through 2 and $50 \mathrm{~km}$ lengths of fiber for measurements made with a $250-\mu \mathrm{m}$ long single-mode MQW-DFB laser at $1.54 \mathrm{mum}$. The parameters $\alpha$ and $\kappa$ were fitted for different fixed values of $\beta_{2}$ from -19 to -21 $\mathrm{ps} / \mathrm{km}^{2}$, and the value of $\beta_{2}$ and corresponding $\alpha$ and $\kappa$ were selected that yielded the minimum quadratic error. This fitting 


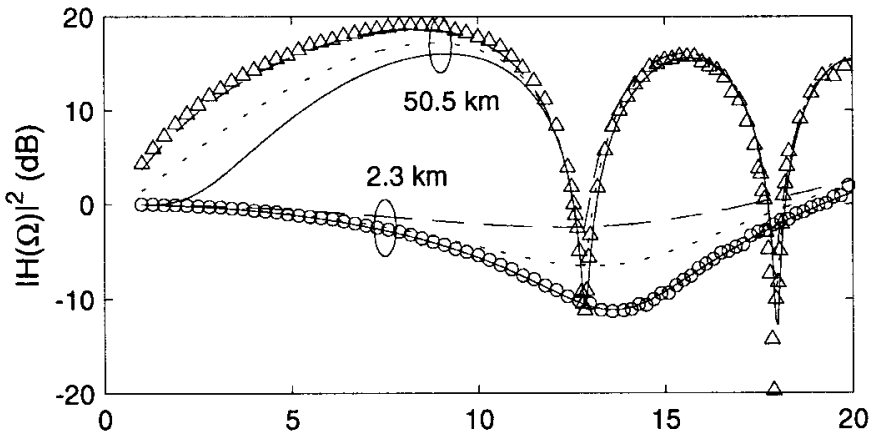

(a)

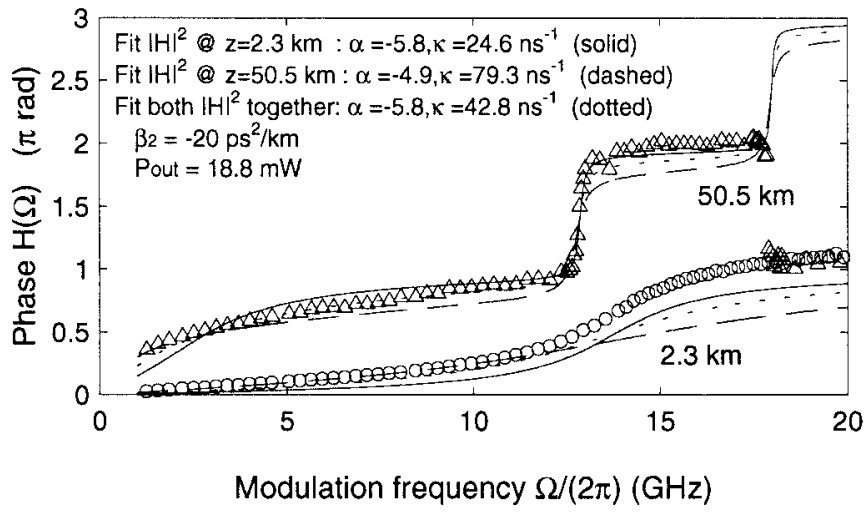

(b)

Fig. 2. Change in modulation response after $2.3 \mathrm{~km}$ (circles) and 50.5 $\mathrm{km}$ (triangles) of standard fiber at $1.54 \mu \mathrm{m}$. The parameters $\kappa$ and $\alpha$ are determined that best fit the change in modulation response after $2.3 \mathrm{~km}$ (solid), $50.5 \mathrm{~km}$ (dashed), and both lengths of fiber (dotted). Output power at laser facet $=18.8 \mathrm{~mW}$.

procedure was not sensitive to the initial values for $\alpha$ and $\kappa$. Fits to each of the experimental curves for different lengths of fiber yield very different values for $\alpha$ and $\kappa$ (see Fig. 2), even if different values of $\beta_{2}$ were used. These values, when used to describe propagation in the other fiber length, produce poor agreement between theory and experiment. We also attempted to find values of $\alpha$ and $\kappa$ that minimize the combined error for several fiber lengths, but no good fits were obtained. ${ }^{1}$

This disagreement is due to the fact that the simple laser model does not fully describe the FM response of the laser. The reason why good fits are obtained for single lengths of fiber is that the value of $\kappa$ mainly determines the width and depth of the dips in $|H(\Omega, z)|^{2}$ that, for small $\kappa$, occur near frequencies $\Omega$ such that:

$$
\theta(\Omega, z) \approx \arctan (-1 / \alpha)+n \pi
$$

${ }^{1}$ For a bulk laser, although a variation of $\kappa$ was also observed, it was not so strong as in the case of this MQW laser. where $n$ is an integer. The fitting procedure tends to adjust the value of $\kappa$ so as to obtain small error near these dips. Since the position in frequency of these dips depends on the fiber length, and $\kappa$ is a function of the modulation frequency, $\Omega$, the fitted value changes for different lengths of fiber. In our experiment, $\kappa$ was mainly determined by the first dip, because higher order dips are narrower, and therefore contained fewer (evenly spaced) data points. (Thus, an estimate for the value of $\kappa$ at a particular modulation frequency can be obtained by choosing a length of fiber, $z$, such that $|H(\Omega, z)|^{2}$ has its first dip at the desired frequency.)

\section{B. Precise Measurement of Laser Chirp}

1) Description of Method: In this section we demonstrate a technique that can conveniently determine the precise laser chirp. In the above described method, a specific expression for the PIR was used to fit for the unknowns. Here, the PIR $(\Omega)$ is directly extracted from the measurement. (Two approaches are considered. The first one uses only the magnitude of the transfer function, $|H(\Omega, z)|^{2}$, whereas the second one uses both magnitude and phase. The second one yields more accurate results but the data reduction is more involved.)

If the value of the dispersion product $\beta_{2} z$ is known, then the only unknowns in (5) are the PIR, which is a complex function of the modulation frequency, and the propagation delay, $\beta_{1} z$. Measurement of $|H(\Omega, z)|^{2}$ for two different lengths of fiber results in two equations that can be solved for the PIR. However, the sign of the imaginary part of the PIR cannot be determined by this procedure.

The PIR, including the sign of the imaginary part, can be extracted from a measurement of the magnitude and phase of $H(\Omega, z)$ and the propagation delay, $\beta_{1} z$, for a single length of fiber. The accuracy of this result can be improved if the $H(\Omega, z)$ for each of several lengths of fiber is measured. Then, at each modulation frequency, $\Omega$, the change in modulation response can be fitted as a function of fiber length to yield real and imaginary parts of $\operatorname{PIR}(\Omega)$ for a fixed value of $\beta_{2}$. The PIR can be determined from measurement of $|H(\Omega, z)|^{2}$ for two lengths of fiber as shown in (8a) and (8b) at the bottom of the page where $\theta_{i}=\theta\left(\Omega, z_{i}\right)=-(1 / 2) \beta_{2} \Omega^{2} z_{i}$ is the dispersion angle after a length $z_{i}$ of fiber. Determination of the PIR using $|H(\Omega, z)|^{2}$ does not require measurement of the propagation delay. The choice of fiber lengths determines at which frequencies a good measurement for $\kappa(\Omega)$ is obtained, since, for a particular length of fiber, $|H(\Omega, z)|^{2}$ is less sensitive to changes in $\kappa(\Omega)$ at frequencies away from the dips.

The fiber lengths can be selected as follows. First, an estimate for $\alpha$ and $\beta_{2}$ is found by using the method described

$$
\begin{aligned}
\operatorname{Re}\{\mathrm{PIR}\} & =-\frac{\alpha}{2}\left(1+\frac{\kappa_{i}}{\Omega}\right) \\
& =\frac{\left|H\left(\Omega, z_{2}\right)\right|^{2} \sin ^{2} \theta_{1}-\left|H\left(\Omega, z_{1}\right)\right|^{2} \sin ^{2} \theta_{2}+\cos ^{2} \theta_{1} \sin ^{2} \theta_{2}-\cos ^{2} \theta_{2} \sin ^{2} \theta_{1}}{4 \sin \theta_{1} \sin \theta_{2}\left(\cos \theta_{1} \sin \theta_{2}-\cos \theta_{2} \sin \theta_{1}\right)} \\
\operatorname{Im}\{\mathrm{PIR}\} & =\frac{\alpha}{2} \frac{\kappa_{r}}{\Omega}= \pm\left[\left|H\left(\Omega, z_{1}\right)\right|^{2}-\left(\cos \theta_{1}+\operatorname{Re}\{\mathrm{PIR}\} \sin \theta_{1}\right)^{2}\right]^{1 / 2} /\left(2 \sin \theta_{1}\right) .
\end{aligned}
$$


in Section A for a single fiber. Using this value for $\alpha$ and (7), the two lengths of fiber can be selected such that the first dips occur near the maximum and minimum frequencies of interest, respectively. However, if the frequency range is too broad, two fibers might not yield sufficiently accurate results, and then several lengths of fiber, not just two, should be used.

Measurement of phase and magnitude of $H(\Omega, z)$ after a single length of fiber yields the PIR directly as long as the propagation delay is known. From (5), we get

$$
\begin{aligned}
& \operatorname{Re}\{\mathrm{PIR}\}=\frac{\cos \theta-\operatorname{Re}\left\{H(\Omega, z) \exp \left(i \beta_{1} \Omega z\right\}\right.}{2 \sin \theta} \\
& \operatorname{Im}\{\mathrm{PIR}\}=-\frac{\operatorname{Im}\left\{H(\Omega, z) \exp \left(i \beta_{1} \Omega z\right\}\right.}{2 \sin \theta} .
\end{aligned}
$$

When the magnitude and phase of $H(\Omega, z)$ is used, the choice of fiber length is not so critical, and the sign of the imaginary part of the PIR can be found. The phase of $H(\Omega, z)$ can be measured simultaneously with the magnitude using a network analyzer. However, the phase depends on the propagation delay. This linear phase term can be extracted using the measurements of $H(\Omega, z)$ as follows. First, the PIR is obtained in an arbitrary frequency range using $|H(\Omega, z)|^{2}$ after two or several lengths of fiber, using the method explained above. This PIR is substituted into (5) to find the theoretical phase of $H(\Omega, z)$ in this frequency range in the absence of propagation delay. The linear phase term added in propagation can be determined by performing a linear fit to the difference of this phase with the experimental phase.

2) Experiment: The same MQW-DFB laser mentioned above and spools of fiber with fiber lengths of $2,4,8$, and 25 $\mathrm{km}$ were used. The values $\alpha \approx-5.5$ and $\beta_{2} \approx-20 \mathrm{ps}^{2} / \mathrm{km}$ were estimated from the simple fittings with single lengths of fiber. In the following figures, the notation in (6) is used together with this estimate for $\alpha$, and the real and imaginary parts of $\kappa(\Omega)$ are displayed, instead of the $\operatorname{PIR}(\Omega)$. This makes it possible to visualize the frequency dependence of $\kappa(\Omega)$, which would otherwise be masked by the strong $1 / \Omega$ term, and also to compare the values for $\kappa$ thus obtained with those of the simple model.

Fig. 3 shows the real (circles) and imaginary (triangles) parts of $\kappa(\Omega)$ obtained from $|H(\Omega, z)|^{2}$ for different combinations of fibers. The measurement is compared with the results using both phase and magnitude (solid and dashed curves, respectively). In Fig. 3(a) and (b), $\kappa(\Omega)$ was obtained from measurement of two fibers. The sets of fiber were selected to yield good results in the frequency range from 3.5 to 8 $\mathrm{GHz}$ and from 4 to $14 \mathrm{GHz}$, respectively. It is observed that the measured $\kappa(\Omega)$ is noisier at low frequencies for the first set, and improves at higher frequencies with respect to the second set. Fig. 3(c) shows the result when four fibers are employed. Above $14 \mathrm{GHz}$ the measurement is noisy because that is the limit set by the shortest fiber that was used. Thus, measurement of $\kappa(\Omega)$ at higher modulation frequencies would require shorter fibers.

Fig. 4 shows the measured $\kappa(\Omega)$ using both phase and magnitude of $H(\Omega, z)$ at several laser output powers. The value of $\kappa(\Omega)$ in Fig. 3(a) was used in a frequency range from 1 to $7 \mathrm{GHz}$ to determine the propagation delay, and

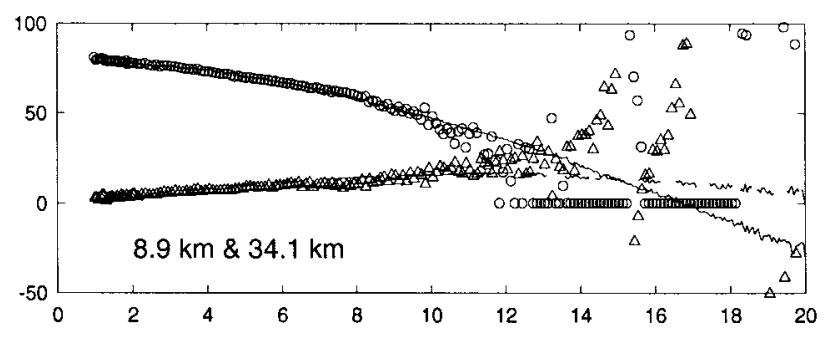

(a)

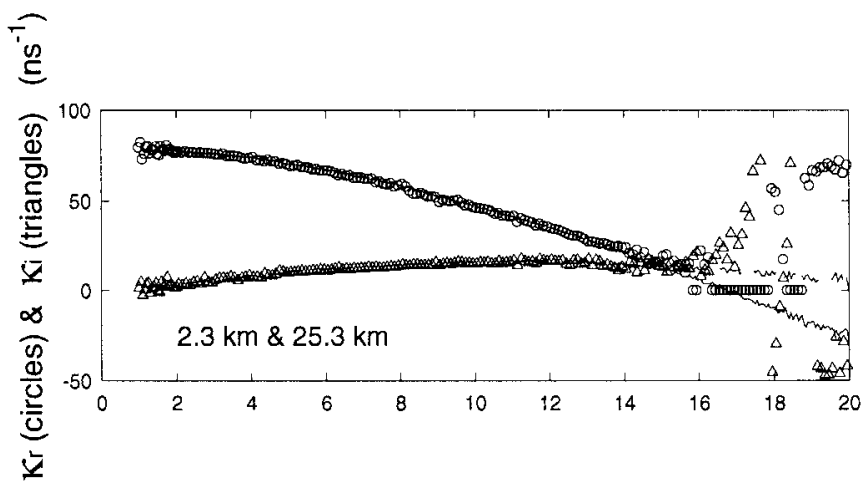

(b)

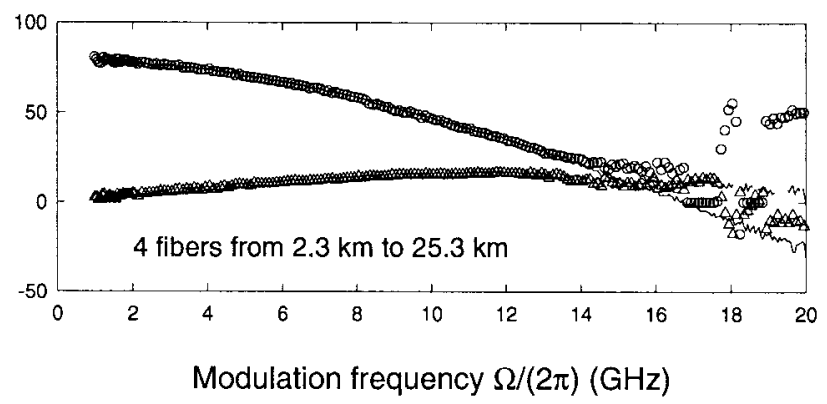

(c)

Fig. 3. Real (circles) and imaginary (triangles) part of $\kappa(\Omega)$ with $\alpha=-5.5$ obtained from $|H(\Omega, z)|^{2}$ for different combinations of fiber. Solid and dashed curves are the result from using magnitude and phase of $H(\Omega, z)$. Output power at laser facet $=18.8 \mathrm{~mW}$.

then the $\operatorname{PIR}(\Omega)$ was extracted from magnitude and phase of $H(\Omega, z)$ after $2 \mathrm{~km}$ of fiber. The variance of the measured PIR for different fibers was very small. At high frequencies, $\kappa_{r}(\Omega)$ becomes negative, which could not be determined from measurement of $|H(\Omega, z)|^{2}$. For the sake of comparison, the simple model would predict constant $\kappa_{r}$ and $\kappa_{i}=0$.

It was observed that $\kappa_{r}(\Omega)$ can be decomposed into a quasifrequency-independent part that increases linearly with output power and a power-independent part, which for this laser is negative (see inset of Fig. 4) and has a strong frequency dependence. The dependence of $\kappa_{i}(\Omega)$ on output power was found to be roughly quadratic. These results can be explained in terms of spatial hole burning and carrier capture models, although a full model that explains the power and frequency dependence of $\kappa(\Omega)$ is beyond the scope of this paper and will be addressed elsewhere.

The measured $\kappa(\Omega)$ in Fig. 4 was used in simulations of propagation through fibers of length 2,11 , and $50 \mathrm{~km}$. Fig. 5 shows the predicted magnitude and phase of $H(\Omega, z)$ (solid) 


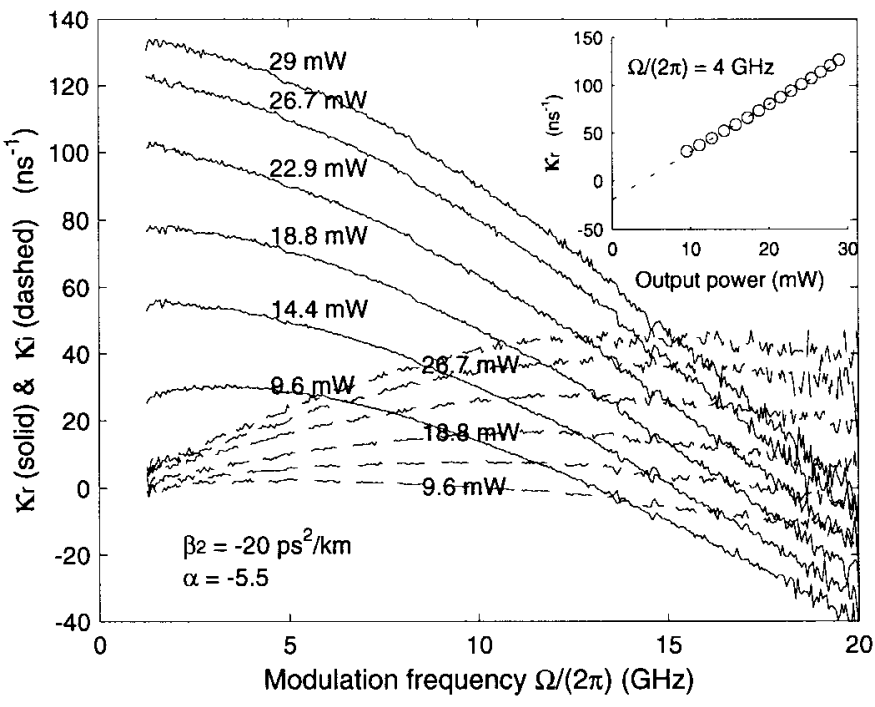

Fig. 4. Real (solid) and imaginary (dashed) part of $\kappa(\Omega)$ with $\alpha=-5.5$ at several laser output powers from magnitude and phase of $H(\Omega, z)$. The inset shows the real part of $\operatorname{kappa}(\Omega)$ as a function laser output power (circles) together with a linear fit (dotted) at $\Omega / 2 \pi=4 \mathrm{GHz}$.

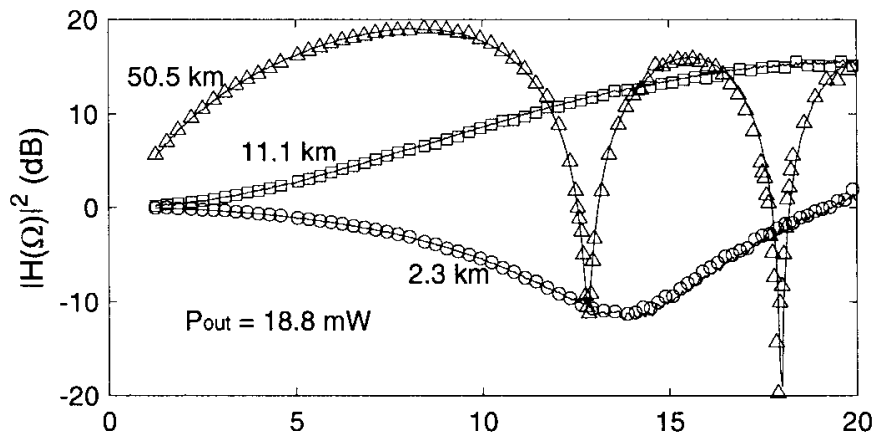

(a)

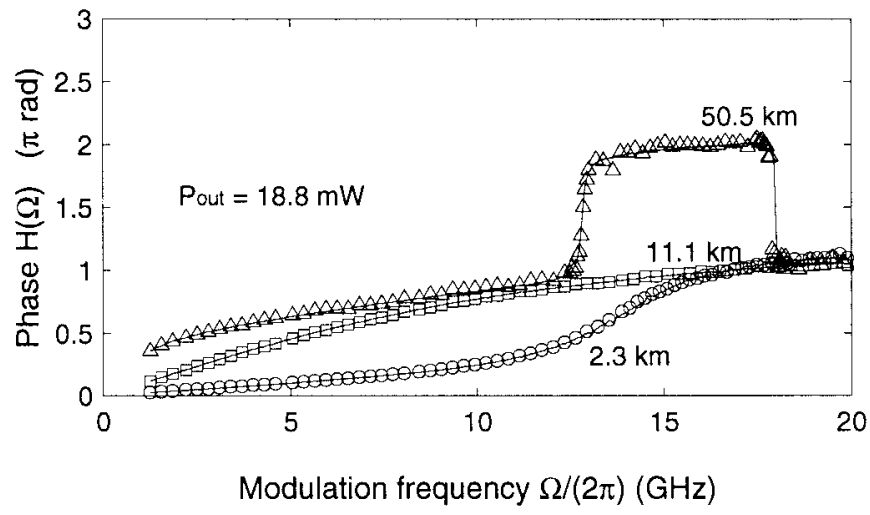

(b)

Fig. 5. Measured change in modulation response after $2.3 \mathrm{~km}$ (circles), 11.1 $\mathrm{km}$ (squares) and $50.5 \mathrm{~km}$ (triangles) of standard fiber together with theoretical curve using frequency dependent $\kappa(\Omega)$. Output power at laser facet $=18.8$ $\mathrm{mW}$.

compared to the experimental points. The two longest fibers had not been used in the original determination of $\kappa(\Omega)$.

In the simple rate equation model, the value of $\kappa_{r}$ is usually assumed to be positive, and the phase of $H(\Omega, z)$ can be shown to be monotonic increasing. However, in real MQW-

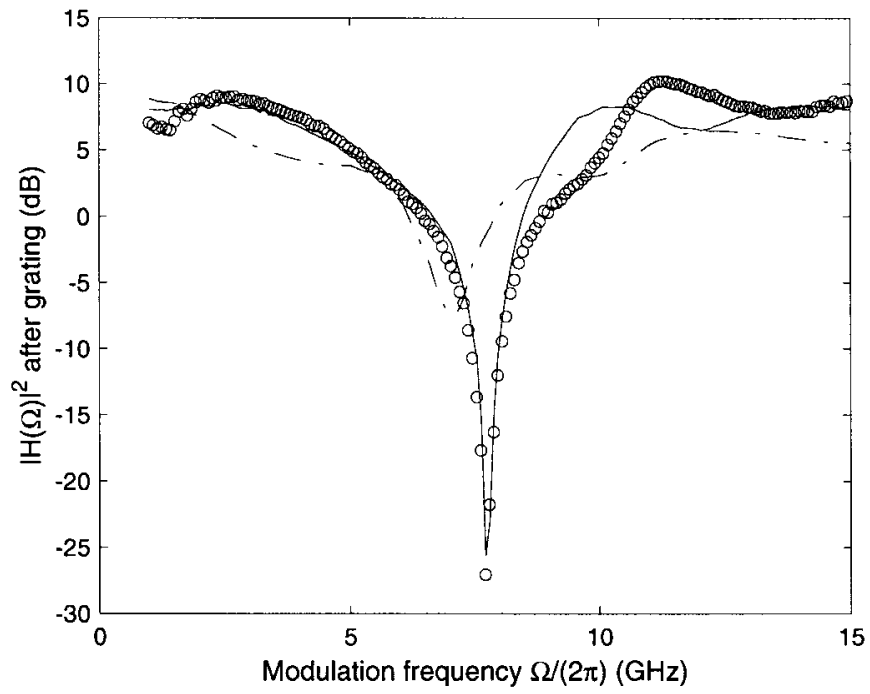

Fig. 6. Baseband transfer function after transmission through fiber grating predicted using frequency dependent $\kappa(\Omega)$ (solid) and constant $\kappa$ (dashed) compared to measurement (circles).

DFB lasers, $\kappa_{r}$ can be negative at some frequencies and the phase of $H(\Omega, z)$ loses monotonicity. In Figs. 2(b) and $5(\mathrm{~b})$, it is observed that the simple model cannot describe the phase of $H(\Omega, z)$ at the frequencies at which $\kappa_{r}$ becomes negative.

\section{Application: Transmission Through Fiber Gratings}

As an example of the implications of the frequency dependence of $\kappa$ in modeling lightwave systems, we consider transmission through fiber gratings. It is known that FM-toIM conversion can be used to advantage to obtain an increase in modulation response [15] and/or decrease in laser relative intensity noise [16]. Given the transmission coefficient of the fiber grating, and a model for the optical source, the separation between Bragg frequency and optical laser frequency that yields optimal performance can be determined.

The baseband transfer function after transmission through the grating is obtained from (3). As an example, transmission through an unchirped fiber grating whose Bragg frequency coincides with the laser frequency will result in no FM-to-IM conversion, because in this case $\tilde{H}\left(\omega_{0}+\Omega\right)=\tilde{H}^{*}\left(\omega_{0}-H\right)$ and therefore $H_{o}(\Omega)=0$.

The amplitude of the transmission coefficient of a fiber grating versus optical wavelength was measured using a tunable laser source and an optical power meter. The phase of the transmission coefficient was determined using a Kramers-Kronig transformation as described in [15]. Using this transmission coefficient, $\tilde{H}(\omega)$, and the previously determined $\kappa(\Omega)$, the baseband transfer function, $H(\Omega)$, was predicted. Fig. 6 shows $H(\Omega)$ predicted by using a frequency dependent $\kappa$ (solid) and a constant $\kappa$ that best fits the change in modulation response after several fiber lengths (dashed) in comparison to the transfer function experimentally measured (circles). Thus, better agreement between simulation and experiment is achieved when the previously determined $\kappa(\Omega)$ is used to describe the FM of the optical transmitter. 


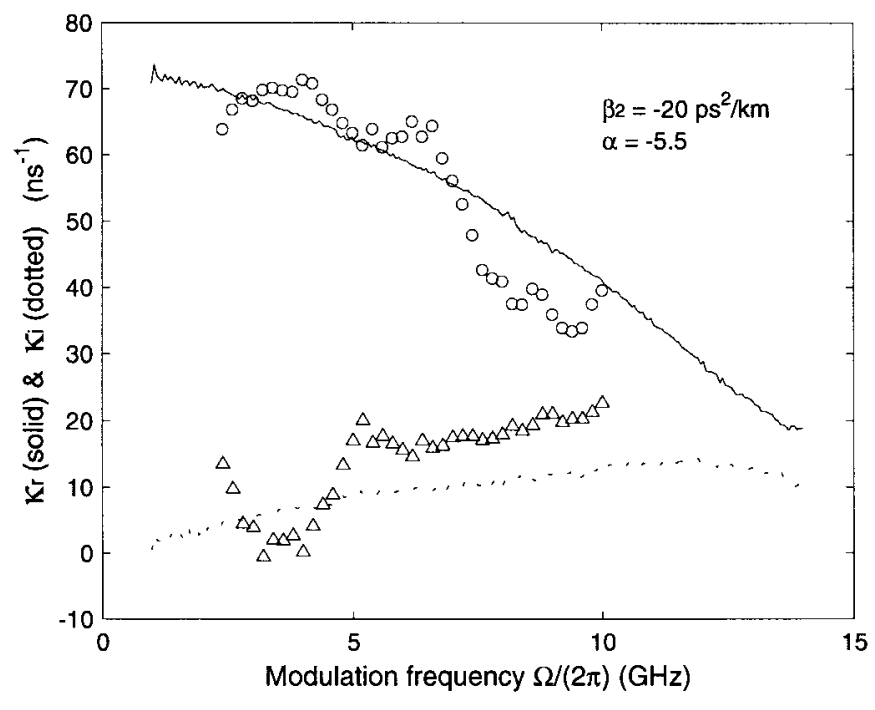

Fig. 7. Real and imaginary part of $\kappa(\Omega)$ obtained from modulation response (solid and dashed, respectively) and from optical spectrum side bands (circles and triangles, respectively). Output power at laser facet $=16.3 \mathrm{~mW}$.

\section{CONCLUSION}

In summary, a new technique has been presented that allows precise determination of the FM response of semiconductor lasers. This response can be compared to models of MQWDFB lasers that account for transverse as well as longitudinal effects to determine which mechanisms contribute to laser chirping, as will be described elsewhere. In addition, performance of lightwave systems that use direct modulation of semiconductor lasers can be better simulated using the experimentally determined laser chirp.

\section{APPENDIX \\ COMPARISON OF MODULATION RESPONSE AND INTERFEROMETER TECHNIQUES FOR DETERMINATION OF LASER CHIRP}

In order to ensure that the measured $\kappa(\Omega)$ was actually an intrinsic laser parameter and was not influenced by other phenomena occurring in transmission through optical fiber, we contrasted it with an interferometer technique.

The power spectrum of the optical field was measured by a scanning Fabry-Perot for a wide range of modulation powers, and the IM index was determined using an electrical spectrum analyzer. As a first approximation, the microwave power can be adjusted so as to obtain known FM indexes. For example, the amplitude of the carrier frequency is approximately zero for a FM index $m_{\mathrm{FM}}=2.4$ [4]. However, the presence of IM complicates the expressions for the power in each of the side bands and this method was not used. Here, $m_{\mathrm{FM}} / m_{\mathrm{IM}}$ and $\varphi_{\mathrm{FM}}-\varphi_{\mathrm{IM}}$ were obtained by fitting the power in the main and first side bands as a function of $m_{\mathrm{IM}}$. From them, we derived $\kappa_{r}(\Omega)$ and $\kappa_{i}(\Omega)$ for a given $\alpha$.

In Fig. 7, the results from the interferometer technique and the modulation response technique have been plotted for comparison. The interferometer technique is noisier, especially at high frequencies, because the microwave source and amplifier we used could only yield a maximum IM index of 0.25. Nevertheless, the same frequency dependence and order of magnitude is observed. Note that in the simple model, $\kappa_{r}$ would be a constant value, and $\kappa_{i}$ would be zero.

\section{ACKNOWLEDGMENT}

The authors are grateful to J. Ianelli and T. R. Chen of the Ortel Corporation for providing the lasers and useful discussions. They also thank A. Bäcker for the careful reading of the manuscript and D. Provenzano for invaluable help in the laboratory.

\section{REFERENCES}

[1] T. L. Koch and R. A. Linke, "Effect of nonlinear gain reduction on semiconductor laser wavelength chirping," Appl. Phys. Lett., vol. 48, no. 10, pp. 613-615, Mar. 1986.

[2] P. Vankwikelberge, G. Morthier, and R. Baets, "CLADISS-A longitudinal multimode model for the analysis of the static, dynamic, and stochastic behavior of diode lasers with distributed feedback," IEEE J. Quatum Electron., vol. 26, no. 10, pp. 1728-1741, Oct. 1990.

[3] R. F. S. Ribeiro, J. R. F. da Rocha, A. V. T. Cartaxo, H. J. A. da Silva, B. Franz, and B. Wedding, "FM response of quatum-well lasers taking into account carrier transport effects," IEEE Photon. Technol. Lett., vol. 7, pp. 857-859, Aug. 1995.

[4] P. Vankwikelberge, F. Buytaert, A. Franchois, R. Baets, P. I. Kuinderma, and C. W. Fredriksz, "Analysis of the carrier-induced FM response of DFB lasers: Theoretical and experimental case studies," IEEE J. Quantum Electron., vol. 25, pp. 2239-2253, Nov. 1989.

[5] W. Huang, X. Li, and T. Makino, "Analytical formulas for modulation responses of semiconductor DFB lasers," IEEE J. Quantum Electron., vol. 31, pp. 842-851, May 1995.

[6] G. Morthier, "An accurate rate-equation description for DFB lasers and some interesting solutions," IEEE J. Quatum Electron., vol. 33, pp. 231-237, Feb. 1997.

[7] J. Kinoshita and K. Matsumoto, "Transient chirping in distributed feedback lasers: Effect of spatial hole-burning along the laser axis," IEEE J. Quantum Electron., vol. 24, pp. 2160-2169, Nov. 1988.

[8] R. Schatz, "Dynamics of spatial hole burning effects in DFB lasers," IEEE J. Quatum Electron., vol. 31, pp. 1981-1993, Nov. 1995.

[9] L. Olofsson and T. G. Brown, "Frequency dependence of the chirp factor in $1.55 \mu \mathrm{m}$ distributed feedback semiconductor laser," IEEE Photon. Technol. Lett., vol. 4, pp. 688-691, July 1991.

[10] L. Bjerkan, A. Royset, L. Hafskjaer, and D. Myhre, "Measurement of laser parameters for simulation of high-speed fiberoptic systems," $J$. Lightwave Technol., vol. 14, pp. 839-850, May 1996.

[11] J. C. Cartledge and R. C. Srinivasan, "Extraction of DFB laser rate equation parameters for system simulation purposes," J. Lightwave Technol., vol. 15, pp. 852-860, May 1997.

[12] B. Wedding, "Analysis of fiber transfer funcion and determination of receiver frequency response for dispersion supported transmission," Electron. Lett., vol. 30, no. 1, pp. 58-59, Jan. 1994.

[13] A. Royset, L. Bjerkan, D. Myhre, and L. Hafskjaer, "Use of dispersive optical fiber for characterization of chirp in semiconductor lasers," Electron. Lett., vol. 30, no. 9, pp. 710-712, Apr. 1994.

[14] R. C. Srinivasan and J. C. Cartledge, "On using fiber transfer functions to characterize laser chirp and fiber dispersion," IEEE Photon. Technol. Lett., vol. 7, pp. 1327-1329, Nov. 1995.

[15] M. McAdams, E. Peral, D. Provenzano, W. K. Marshall, and A. Yariv, "Improved laser modulation response by frequency modulation to amplitude modulation conversion in transmission through a fiber grating," Appl. Phys. Lett., vol. 71, no. 7, pp. 879-881, Aug. 1997.

[16] M. McAdams, D. Provenzano, E. Peral, W. K. Marshall, and A. Yariv, "Effect of transmission through fiber gratings on semiconductor laser intensity noise," Appl. Phys. Lett., vol. 23, no. 8, pp. 3341-3343, Dec. 1997. 


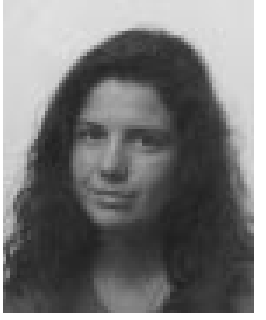

Eva Peral (M'98) was born on November 23, 1972 in Granada, Spain. She received the degree of Ingeniero Superior de Telecomunicaciones with Honors from the Universidad Politecnica de Valencia (UPV), Spain, in 1995. She began her doctoral studies with the Optical Communications and Photonic Engineering Group in UPV and is currently working towards the Ph.D. degree at the California Institute of Technology, Pasadena, CA.

During 1995, she was a visiting student in University of Southampton, Glasgow, Scotland, and collaborated with the Optoelectronics Group where she was engaged in integrated optics. She has worked for Philips, Valencia, Spain, and MercedesBenz, Sindelfingen, Germany. Her research interests include semiconductor lasers, fiber gratings, nonlinear phenomena in optical communications and integrated optics.

Ms. Peral received the National Award for Academic Excellency from the Spanish Ministry of Culture and Education in 1996.

William K. Marshall received the Ph.D. degree in applied physics from the California Institute of Technology, Pasadena, CA, in 1997.

$\mathrm{He}$ is currently a Postdoctoral student at the same institution. His current research interests center on issues arising from interplay of the dynamical properties of semiconductor lasers with the effects of propagation in optical fibers and lightwave systems. Other research topics have included semiconductor lasers and laser arrays, waveguide modulators, and applications of free-space optical communications to deep-space exploration. Previously, he was a Member of the Technical Staff in the Optical Communications Group at the Jet Propulsion Laboratory, Pasadena.

Dr. Marshall is a member of the Optical Society of America (OSA).
Amnon Yariv (S'56-M'59-F70-LF'95) was born in Tel Aviv, Israel. He received the B.S., M.S., and Ph.D. degrees in electrical engineering from the University of California, Berkeley, in 1954, 1956, and 1958, respectively.

After graduating from Geulah High School, Tel Aviv, he served in the first artillery unit of the Israeli Defense Forces during the 1948-1950 war of independence. In 1959, he joined Bell Telephone Laboratories, Murray Hill, NJ, where he worked on the early stages of the laser effort. In 1964, he joined the California Institute of Technology, Pasadena, as an Associate Professor of Electrical Engineering and became Professor in 1966. In 1980, he became the Thomas G. Myers Professor of Electrical Engineering and in 1996, he became the Martin and Eileen Summerfield Professor of Applied Physics. He has taken part (along with various co-workers) in the discovery of a number of early solid-state laser systems in proposing and demonstrating the field of semiconductor integrated optics, the invention of the semiconductor distributed feedback lasers and in pioneering the field of phase conjugate optics. His present research efforts are in the areas of nonlinear optics, semiconductor lasers, and integrated optics with emphasis on communication and computation. He has published widely in the laser and optics fields and has written a number of basic texts in quantum electronics, optics, and quantum mechanics. He is Founder and Chairman of the Board of the ORTEL Corporation.

Dr. Yariv is a member of the American Physical Society, Phi Beta Kappa, the American Academies of Arts and Sciences, the National Academies of Engineering and Sciences, and a Fellow of the Optical Society of America (OSA). He was the recipient of the 1980 Quantum Electronics Award of the IEEE, the 1985 University of Pennsylvania Pender Award, the 1986 Optical Society of America Ives Medal, and the 1992 Harvey Prize. 